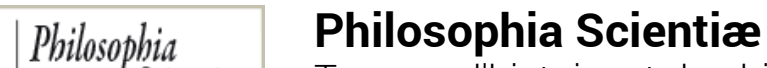

Scientie Travaux d'histoire et de philosophie des sciences

14-2 | 2010

Louis Rougier, De Torricelli à Pascal

- Chapitre IV - Les expériences de Rouen

Louis Rougier

(2) OpenEdition

Journals

Édition électronique

URL : https://journals.openedition.org/philosophiascientiae/180

DOI : 10.4000/philosophiascientiae.180

ISSN : 1775-4283

Éditeur

Éditions Kimé

Édition imprimée

Date de publication : 1 octobre 2010

Pagination : 80-101

ISBN : 978-2-84174-536-4

ISSN : 1281-2463

Référence électronique

Louis Rougier, " - Chapitre IV - Les expériences de Rouen », Philosophia Scientiæ [En ligne], 14-2 |

2010, mis en ligne le 01 octobre 2013, consulté le 21 septembre 2021. URL : http://

journals.openedition.org/philosophiascientiae/180 ; DOI : https://doi.org/10.4000/

philosophiascientiae. 180

Tous droits réservés 


\section{- Chapitre IV - Les expériences de Rouen}

\section{1 - Les insuccès de Mersenne et la réussite de Petit}

Au début de l'été 1644, Mersenne venait d'achever ses Cogitata et commençait la rédaction de ses Reflexiones, lorsqu'il reçut de Michel-Ange Ricci la copie des deux lettres de Torricelli. Celui-ci n'était nullement un inconnu pour le Minime : ils étaient en correspondance depuis 1643 et échangèrent de nombreuses lettres au cours de cette année 1644 et par la suite sur des questions de géométrie, d'acoustique, d'optique, sans qu'il soit jamais question des expériences sur le vide ${ }^{34}$.

Au premier chapitre des Reflexiones, où il consignait au jour le jour des idées en matière scientifique, Mersenne note qu'aux sept constatations en faveur du vide que l'on trouve dans Héron de Byzance, il y a lieu d'en joindre une huitième, qu'on vient d'obtenir à Florence, au moyen d'un tube de verre rempli de mercure. Il consigne l'objection que Mersenne s'était faite à lui-même contre l'hypothèse de la colonne d'air : elle implique que l'air des régions hautes n'ait pas la même densité que celui des couches inférieures. Au chapitre II, il écrit que l'expérience contribuera à établir que la nature n'est pas partout identique à elle-même. Il entame une discussion sur l'essence de la pesanteur. On ne pourra se prononcer sur la cause réelle du phénomène tant qu'on ne saura pas si le haut du tube est réellement vide : pour cela, il conviendrait d'examiner si le son et la lumière s'y propagent — idée que reprendront Huygens et Roberval - et si la vie s'y maintient. En tous cas, la question est d'importance et le philosophe qui en donnera la solution l'emportera sur ses rivaux.

Pour son compte, curieux de toute expérience nouvelle, il tente de refaire celle de Florence. Il n'y avait point réussi quand, délivré de l'impression de son gros volume, les Cogitata physico-mathematica, n'ayant plus rien qui le retînt dans la capitale depuis le départ de M. Descartes, il se mit en route, fin octobre, pour un voyage de huit ou neuf mois qu'il projetait en Italie.

À Rome, il vit Lucas Halstenius, bibliothécaire du Vatican, qui lui parla d'un capucin milanais, Valeriano Magni, homme savant et original. Mersenne s'en fut le voir : «C'est un vaste et vaillant esprit », écrit-il à Hevelius. Le 24 décembre, il prend congé d'Halstenius, et, quelques jours après, se trouve à Florence, où Torricelli lui montre les tubes, refait ses

34. Lettere di Evangelista Torricelli al P. Marin. 
expériences devant lui et lui expose sa théorie. En juillet 1645, Mersenne est à Paris. Il rapporte des copies plus complètes des lettres de Torricelli à Ricci, des renseignements détaillés de la bouche même de l'inventeur sur la conduite de l'expérience. Il reprend ses essais, mais échoue à nouveau. Roberval, qui nous conte ces détails, attribue ces échecs à la difficulté de se procurer à Paris des tubes convenables. Mersenne interrompt peu après ses tentatives pour aller passer l'hiver de 1645-1646 dans le midi. Il ne revint, nous dit Baillet, qu'au commencement de septembre 1646. De retour, il se remet à l'œuvre avec Chanut, ambassadeur de Suède, de passage à Paris. Nouvel échec. Renonçant à se procurer à Paris le matériel convenable, il estime que la verrerie de Rouen peut seule lui fournir de bons tubes.

C'est à l'ingénieur Petit, intendant aux fortifications, réputé le plus habile expérimentateur du royaume, qu'il semble s'être adressé. Précisément, celui-ci, au mois d'octobre 1646, se rendait à Dieppe pour s'assurer si, comme l'en assurait depuis cinq ans un inventeur marseillais, on pouvait descendre au fond de la mer et y demeurer pendant cinq à six heures avec une machine. À l'aller, Petit s'arrêta à Rouen et informa les deux amis qu'y comptait Mersenne, Étienne Pascal et son fils, de l'expérience d'Italie et de la nécessité d'avoir de bons tubes pour la réussir. On convint qu'à son retour de Dieppe, tous trois tenteraient l'expérience. C'est ainsi que Petit, en présence d'Étienne et de Blaise Pascal, plus de deux années après la lettre de Ricci à Mersenne, fut le premier en France, au dire de Pierius, à reproduire, dans le courant du mois d'octobre 1646, l'expérience de Torricelli.

Tous trois s'étonnèrent d'abord de ce vide qui paraissait au-dessus du vif-argent, dans le haut du tube. La nature n'en aurait-elle pas horreur, comme l'enseignait l'École? Bien plus, le vide apparent devenait plus grand ou plus petit, selon que l'on haussait ou baissait le tube, sans retirer de la cuvette l'extrémité qui y trempait. Enfin, ayant versé de l'eau sur le vif-argent de la cuvette, en soulevant le tube jusqu'à ce que l'extrémité ouverte se trouvât au niveau de la couche d'eau, ils virent avec étonnement celle-ci monter jusqu'au haut du tube, le remplir en son entier en se mêlant au vif-argent pendant quelques instants, puis expulser le vif-argent, si bien que le tube se trouvait finalement rempli d'eau uniquement, y compris l'espace vide laissé par le mercure.

Sur le conseil de Mersenne, Petit écrivit à Chanut, de retour de Stockholm, pour lui annoncer le succès de l'expérience et toutes les particularités signalées. Dans l'autographe de cette lettre, qui existe encore, on peut lire : «C'est de l'expérience de Torricelli touchant le vide, dont je veux vous entretenir. » Cette lettre est de novembre 1646 et plusieurs 
copies en furent distribuées en France. Si elle ne fut pas alors imprimée - elle le fut par la suite - comme le véritable procès-verbal de l'expérience, «c'est, raconte Petit, qu'un de ses intimes, qui avait beaucoup enchéri par-dessus les premières observations, et qui avait quantité de choses à dire, traiterait tout cela dignement et à fond ». Cet intime était Blaise Pascal.

\section{2 - Les expériences de Blaise Pascal}

Quelle fut l'attitude de Pascal en présence de l'expérience de Petit? Tout d'abord, il fit des objections. L'espace lui paraissait vide, l'était-il en réalité? L'air, plutôt que de laisser un vide se produire, n'aurait-il pas pénétré par les pores du verre? À quoi Petit répliquait : pourquoi l'air ne pénètre-t-il pas davantage, en sorte à expulser tout le vif-argent? En outre, le verre n'est point perméable à l'air. Il y avait lieu toutefois de se demander si une petite parcelle d'air, fût-elle grosse comme une tête d'épingle, n'était pas demeurée au fond du tuyau, ou entrée avec le vif-argent lorsqu'on le versait, ou apportée avec le doigt, lorsqu'on l'apposait sur l'extrémité ouverte. Une de leurs premières pensées fut aussi que, si le vif-argent ne descendait pas plus bas, c'est que la nature ne pouvait souffrir un plus grand vide, ou, dans l'hypothèse de l'inexistence du vide, une plus grande raréfaction. Ils renoncèrent vite à cette dernière explication : en effet, que l'on haussât ou abaissât le tuyau, la hauteur du vif-argent restait la même, encore que le vide apparent augmentât ou diminuât.

Pour en avoir le cœur net, Pascal imagina de nombreuses expériences « très différentes de celle-là », où il fit varier les conditions du phénomène, se servant de liqueurs de densité différente, d'eau, d'huile, de vin, etc., et même d'air, et en employant tour à tour des tubes de toutes longueurs et dimensions. En outre, comme pour provoquer de fructueuses objections, il fit ses nouvelles expériences devant plus de cinq cents personnes de toutes conditions, entre autres devant cinq ou six pères jésuites du Collège de Rouen et un savant de ses amis, Adrien Auzoult, qui envoya une relation à Gassendi.

La conclusion de Pascal fut «qu'un vase ou vaisseau, si grand qu'on pourra le faire, peut être rendu vide de toutes les matières connues en la nature et qui tombent sous nos sens ». Cela se passait à la fin de 1647 et dans les deux premiers mois de l'année suivante.

Le résultat, auquel parvint Pascal, manifeste bien que lui et ses amis virent avant tout dans l'expérience de Torricelli la preuve de la possibilité 
du vide. C'est le haut du tube qui accapare uniquement leur attention : le vaccum apparent est-il plein ou vide, voilà qui passionne les esprits.

Le camp des péripatéticiens, d'abord interdit, eut vite fait de se ressaisir. Jacques Pierius, curé de Villède, docteur en médecine et en théologie, professeur de philosophie au Collège que l'archevêque de Rouen venait de fonder en concurrence de celui des Jésuites, rédigea en vingtquatre heures une dissertation, An detur vacuum in rerum natura, pour démontrer que la thèse péripatéticienne demeurait intacte. Le vide n'existe pas; les anges eux-mêmes ne sauraient le réaliser, car ils ne disposent que d'une puissance finie, et la répugnance de la nature au vide est illimitée; Dieu seul pourrait le faire, s'il avait dessein d'exterminer le monde. La partie supérieure du tube n'est vide qu'en apparence : elle est remplie de vapeurs de mercure. En quoi Pierius ne se trompe pas, mais il en donne une raison purement aristotélicienne. Le mercure est un corps bâtard, qui, n'ayant pas encore reçu sa forme substantielle, ne sait quel est son mouvement naturel, s'il doit monter, descendre ou girer, et qui possède la particularité de se raréfier spontanément comme en témoigne la colique des peintres qui emploient le blanc de céruse. Effrayée par cette instabilité qui met en péril l'ordre universel, la nature intervient : dans le haut du tube, elle développe « une force [mot illisible] » qui arrache au mercure des vapeurs pour écarter tout danger de vide. Quand le mercure n'en peut plus fournir, il s'arrête et demeure suspendu à ses propres vapeurs. L'humidité du mercure, du vin et de l'eau explique du reste qu'il se produise une émission de vapeurs dans le haut du tube barométrique.

Le haut du tube est plein d'air raréfié; le haut du tube est plein des vapeurs du mercure, voilà ce qu'on objectait, avec raison, contre le vide. Pascal institue, vers les mois de janvier et février 1647, une série d'expériences pour y répondre. Il établit d'abord que la hauteur du liquide demeure la même, de quelque manière qu'on fasse varier la chambre barométrique, en soulevant ou en inclinant le tube. Si on introduit une bulle d'air, le niveau du mercure baisse un peu, mais la quantité d'air introduite a seule une influence sensible sur le phénomène, indépendamment de l'espace laissé libre pour la raréfaction de l'air. Pour réfuter Pierius, Pascal dresse un mât dans une cour de la verrerie de Rouen. Il y attache deux tubes de quarante pieds. Ayant convié ses contradicteurs à une conférence solennelle, il les somme de lui dire ce qui va se passer, si on refait l'expérience du vide avec ces deux tubes, l'un étant rempli d'eau, l'autre de vin. Le vin étant plus spiritueux que l'eau, la colonne de vin fournira plus de vapeurs que la colonne d'eau et devra descendre davantage. L'expérience, qui prouve seulement que la hauteur 
du liquide n'est pas en raison inverse de sa volatilité, dans les conditions où il l'entreprit, lui donne apparemment raison.

\section{3 - Perplexités théoriques suscitées par leur interpré- tation}

Ces expériences eurent un grand retentissement à Paris. Elles devinrent l'entretien favori dans [le monde?] savant pendant l'hiver. Le Sieur de Saint-Ange, capucin, raconta à Rouen, en 1647 « qu'il avait entendu parler de cette expérience à Paris devant que de venir en cette ville, en une compagnie où on avait fait un grand état dudit Sieur Pascal ${ }^{35} »$. Cette compagnie était-elle celle de l'abbé Bourdelot ${ }^{36}$, fondée en 1638, où fréquentaient le P. Mersenne, Gassendi, La Mothe ${ }^{37}$, Le Pailleur, Petit, Pascal père, Auzoult, Pecquet, Roberval, et d'autres encore? Il n'importe. Les problèmes théoriques, suscités par l'observation de Galilée et l'expérience de Torricelli, dont l'attention avait été détournée par suite de l'insuccès des tentatives de Mersenne, se posent avec une acuité toute nouvelle aux curieux et savants de l'époque.

Ces problèmes, Gassendi les énumère dans une sorte de questionnaire qu'il rédige, sitôt au fait par Adrien Auzoult des expériences de Pascal.

En premier lieu, cet espace qui demeure au-dessus du niveau du mercure (ou de l'eau) après sa dépression, doit-il être considéré comme purement vide? Ensuite, ce vide, si petit soit-il, comment s'expliquer que la nature, qui manifeste pour lui, dans les autres cas, une si grande répugnance, puisse le tolérer? En troisième lieu, quelle force empêche le mercure de s'écouler entièrement et le maintient à une certaine hauteur (de même pour l'eau)? Enfin, d'où vient l'impétuosité avec laquelle, soit l'air, soit l'eau, lorsqu'on soulève l'orifice du tube au niveau de l'air ou de l'eau, se précipite dans le tube, pousse le mercure, et, ayant affirmé sa supériorité, sort après l'avoir chassé ${ }^{38}$ ?

En réponse à la première question, Roberval inclinait à admettre le vide. $\mathrm{Si}$, comme le prétendaient les scolastiques, le mercure est soutenu par des vapeurs ou par de l'air raréfié, sa hauteur doit être proportionnelle

35. Cousin, Bibliothèque de l'École des Chartes, 1842-1843, t. IV, p. 119.

36. Le Gallois, Conversations de M. l'Abbé Bourdelot, etc., Paris 1672.

37. [NdE - La Mothe le Vayer.]

38. De nupero experimento circa vacuum, ap. Opera omnia, t. L, 204, col. I. 
à la quantité de ces vapeurs ou de cet air, par conséquent à la surface de volatilisation du liquide et à la surface d'absorption du tube ; enfin, il doit y avoir un rapport constant entre les dimensions de l'espace inoccupé et le poids de la colonne suspendue. Avec des tubes de formes, de longueurs et de diamètres variés, Roberval fait varier toutes ces données et montre qu'elles n'ont pas d'influence sur la hauteur du mercure, qui demeure constante. Il réalise également l'expérience suivante : si on laisse pénétrer une bulle d'air dans le tube où on a fait le vide et si on le retourne brusquement, après avoir obturé l'extrémité ouverte avec le doigt, le mercure vient occuper le fond du tube où se trouvait l'espace vide qui se trouve transporté instantanément à l'extrémité bouchée par le doigt, cependant que la bulle d'air monte plus lentement à travers le mercure. Il en est de même, lorsqu'on opère avec une goutte d'eau ${ }^{39}$.

Conclura-t-on que la chambre barométrique est vide? Mais alors, comment la lumière peut-elle la traverser? Admet-on une théorie corpusculaire de la lumière, comme Gassendi et les atomistes : alors, le vacuum n'est plus vide, puisque des particules lumineuses le traversent, et voilà Gassendi, qui n'ose assurer que la chambre barométrique soit entièrement vide. Admettra-t-on, comme Huygens, une théorie ondulatoire de la lumière, la transmission des rayons lumineux dans le haut du tube « prouve qu'une matière différente de l'air se trouve dans le tuyau, et que cette matière doit avoir percé le verre, ou le vif-argent, ou l'un et l'autre, qui sont tous deux impénétrables à l'air ${ }^{40} »$. À cette difficulté venant de la propagation de la lumière, Mersenne ajoute celle qu'offrirait la propagation du son. Il propose, dans ses Reflexiones, d'enfermer dans l'espace libre du tube une mouche, un oiseau, même un homme muni d'un marteau qui lui permettrait de briser sa prison en cas de danger, ou, à défaut d'un être humain, un chat ou n'importe quel animal qui manifesterait par ses cris et ses mouvements ce qui se passe dans le vacuum.

Quelle est la cause de la suspension du liquide? Torricelli prétendait que c'était la pression de la colonne d'air dans le mercure de la cuvette. Roberval multiplie contre la colonne les objections, et Mersenne, sous son influence, rédige le vingt-troisième chapitre des Reflexiones, dont le sommaire annonce de «nombreuses objections contre la colonne d'air ».

L'objection la plus grave était celle que Torricelli s'était faite à luimême : étant données la hauteur et la densité, qu'on croyait connaître, de l'atmosphère, la colonne de mercure soulevée devrait être bien plus

39. Ces expériences sont consignées dans la lettre de Roberval à Desnoyers, du 20 septembre 1647.

40. Huygens, Traité de la lumière, Leyde, 1890, p. 10-11. 
haute. Le Florentin s'en tirait en supposant que l'atmosphère va en se raréfiant dans les colonnes supérieures. Mais Roberval estimait de mauvaise méthode d'écarter par une hypothèse nouvelle une objection à une hypothèse. Pour lui, les observations sur la durée du crépuscule et sur la réfraction étaient trop probantes pour qu'il consente à abaisser audessous de cinquante milles la hauteur de l'atmosphère. À ce taux, il fallait admettre une raréfaction de l'air dans les régions d'en-haut bien supérieure à celle que semblaient lui permettre d'accepter des expériences réalisées par lui en 1643 : il croyait avoir démontré, alors, que l'air ne pouvait être dilaté que jusqu'à occuper soixante-dix fois son volume. Victime du dogme traditionnel de la passivité des substances, Roberval, bien qu'il en eût aux aristotéliciens, se demandait quelle force eût pu produire une telle dilatation. Quelle autre force que la chaleur? Mais ne sait-on pas que la chaleur diminue à mesure qu'on s'élève. L'idée ne lui venait pas que l'air pût spontanément se raréfier.

Contre l'hypothèse de la colonne, Roberval avait d'autres raisons, plus théoriques qu'expérimentales, tirées de ses conceptions cosmologiques, exposées en 1642 dans son Aristarque, réimprimées en 1643, et finalement, insérées dans les Reflexiones de Mersenne. Selon lui, l'air n'agit pas seulement par son propre poids, mais comme véhicule de l'attraction, force mystérieuse et formidable, qui tient agrégées les parties de chaque système planétaire. Pour produire un grand vide, il faudrait soulever tout le système terrestre : théoriquement, cela est possible, mais les hommes ne disposent d'aucune force capable de faire un vide d'un pied cube. Comment imaginer, dès lors, que vingt-sept pouces de mercure fassent contrepoids à cette force?

Si ce n'est pas une pression extérieure qui soulève le mercure, c'est une attraction intérieure qui l'attire. Mais comment la concevoir? Est-ce l'horreur du vide? Torricelli a déjà réfuté cette explication : cette force devrait être proportionnelle au volume de la chambre barométrique, ce que contredit l'expérience. Et puis, comment expliquer la violence avec laquelle l'eau fait irruption dans le tube, jusqu'à le briser, quand on en introduit dans la cuve, et les oscillations du mercure qui monte et descend une douzaine de fois avant de prendre son niveau?

\section{4 - Pascal devancé : la Dissertation et la Justification du P. Magni}

Pascal, dont la santé était ébranlée, quitta Rouen à la fin de mai 1647 pour s'installer à Paris avec sa sœur Jacqueline. Il était en train de rédiger son Traité sur le vide, où devaient être relatées les expériences de 
Rouen et l'interprétation théorique qu'il en proposait, lorsque, dans le courant de l'été 1647, Roberval reçut une lettre de Des Noyers, secrétaire de la Reine de Pologne, lui mandant qu'un capucin italien, supérieur des missions apostoliques du Nord, celui-là même que Mersenne avait rencontré à Rome en 1644, venait de faire, à Varsovie, dans le courant de juillet 1647, une conférence d'apparat où il avait montré un espace absolument vide, à l'aide d'un tube rempli de vif-argent. La dissertation où Magni exposait son expérience suivit de près la missive de Des Noyers. Mersenne en reçut un exemplaire, sous ce titre Demonstratio ocularis, loci sine locato, corporis successive moti in vacuo, luminis nulli corpori inhaerentis. L'approbation est datée du 16 juillet 1647 et l'auteur consigne qu'il a achevé sa narration le 12 juillet.

L'expérience de Varsovie est bien celle d'Italie : elle est postérieure de six mois aux conférences de Rouen, mais antérieure de trois mois à la publication des Expériences nouvelles de Pascal. Alors que Torricelli appréciait son expérience, moins pour le vide qu'elle produisait que pour la pesanteur de l'air qu'elle démontrait, Magni, comme Pascal, ne prête attention qu'au vide qu'elle manifeste, au vide qui convainc d'erreur Aristote, en montrant un lieu sans corps localisé; un espace vide où le mouvement se produit, des qualités, telle que la lumière, sans substance à laquelle elles sont inhérentes. Rejeter la théorie du lieu, celle du mouvement, celle de la substance, c'est rejeter toute l'ontologie et la dynamique du Lycée. Le tube barométrique brandi par Magni, c'est la flèche du Parthe dans le système du Stagirite.

Roberval répondit à Des Noyers, le 20 septembre 1647. Il reproche à Magni d'avoir manqué de délicatesse, non à l'adresse de Pascal, mais à l'égard de Torricelli. Il a en mains la lettre de Torricelli que Ricci communiqua à Mersenne; il rappelle les essais infructueux du Minime pour réaliser l'expérience du vide et son voyage en Italie. Il insiste sur le succès des expériences de Rouen, sans insinuer que Magni ait pu en avoir connaissance. Il expose ses propres recherches et son propre sentiment qui l'incline à croire que le haut du tube est effectivement vide d'air, mais il ne saurait l'affirmer avant d'avoir réalisé certaines expériences qui lui montreront si la vie est possible.

La réponse de Magni ne se fit pas attendre. Elle est contenue dans une brochure datée de Varsovie, le 5 novembre 1647, sous ce titre : De inventione artis exhibendi Vacuum narratio Apologetica Valeriani Magni... ad nobilem et clarissimum virum P. de Roberval. Il y retrace la genèse de son expérience.

En Italie, il n'a entendu parler que de l'observation des fontainiers de Florence. Nulle part, ni à Rome, ni à Florence, il n'a eu vent de 
l'expérience de Torricelli et de sa lettre à Ricci : il ignorait jusqu'aux noms de ces deux personnages. Il reconnaît avoir reçu à Rome la visite de Mersenne, mais sans que celui-ci lui ait fait la moindre confidence à ce sujet. Ce sont ses propres réflexions qui l'ont amené à ces résultats. Étant à Cracovie en 1644, un mathématicien, Titus Livius Buratinus, lui fit présent d'une balance hydrostatique, à l'aide de laquelle il chercha les densités comparatives de l'eau et du mercure. Il lui vint naturellement à l'idée de vérifier le résultat obtenu, à savoir le rapport de 1 à 14 , en reproduisant avec le mercure le phénomène dont les puisatiers de Florence avaient demandé l'explication à Galilée. Si la hauteur limite de la colonne d'eau soulevée est de 32 pieds, elle sera quatorze fois moindre pour le mercure. Il imagine ainsi l'expérience barométrique, mais, manquant de tubes de verre, il recourt à des tuyaux de bois et perd sa peine. Cependant, un célèbre verrier vénitien, Gaspard Brunorius, lui ayant fourni des tubes de diverses longueurs, il réussit son expérience vers le mois de juin ou juillet 1647 et la recommença publiquement le 18 juillet de la même année.

Rien ne nous autorise à suspecter l'exactitude du récit de Magni. Il cite à sa décharge le témoignage de ceux qui l'ont lu et loué sans relever la priorité de Torricelli. Eût-il adressé sa dissertation à Mersenne, si celui-ci l'avait entrepris à Rome sur l'expérience du Florentin? Quand Pascal, dans sa lettre à Ribeyre, déclara qu'en 1647 il ignorait que l'auteur de l'expérience d'Italie fût Torricelli, ses défenseurs le déclarent de bonne foi, alors que ses intimes, Petit, Mersenne, Roberval savaient à cette époque parfaitement à quoi s'en tenir. Pourquoi, au nom d'une bien plus grande vraisemblance, refuser toute confiance au P. Magni? En tous cas, Torricelli, à part sa lettre privée à Ricci, n'ayant rien publié touchant l'expérience barométrique, le Capucin est fondé à prétendre qu'il est le premier à la présenter au public dans un livre imprimé. Ce n'était point pour se concilier les bonnes grâces de Blaise Pascal qui, maladivement susceptible, se trouvait ainsi prévenu et supportait malaisément la concurrence.

\section{5 - Pascal partisan de Galilée : les expériences nouvelles touchant le vide}

Bien que Pascal fût informé de la Dissertation et de l'Apologie du P. Magni, délaissant pour le moment son grand Traité du vide, il se hâta de rédiger un petit Abrégé de ses expériences de Rouen, parce que, écritil, « les ayant faites avec beaucoup de frais, de peine et de temps, j'ai craint qu'un autre qui n'y aurait employé le temps, l'argent ni la peine, 
me prévenant, ne donnât au public des choses qu'il n'aurait pas vues, et lesquelles par conséquent il ne pourrait pas rapporter avec l'exactitude et l'ordre nécessaire pour les déduire comme il faut : n'y ayant personne qui ait eu des tuyaux et des siphons de la longueur des miens et peu qui voulussent se donner la peine nécessaire pour en avoir $\gg$. Son dessein n'est pas seulement d'articuler une revendication de priorité, il veut faire œuvre de probité, en départageant ce qui lui appartient de ce dont il n'est point l'inventeur :

Vous approuverez [sans doute ${ }^{41}$ ] que je me défende également, et de ceux qui voudraient m'ôter quelques-unes des expériences que je vous donne ici, et que je vous promets dans mon traité entier, puisqu'elles sont de mon invention; et de ceux qui m'attribueraient celle d'Italie, dont je vous ai parlé, puisqu'elle n'en est point.

Le permis d'imprimer l'ouvrage est du 8 octobre 1647 et il parut sous le titre : Expériences nouvelles touchant le vide faite dans des tuyaux, seringues, soufflets et siphons de plusieurs longueurs et figures, avec diverses liqueurs, comme vif-argent, eau, vin, huile, air, etc.; avec un discours sur le même sujet, où est montré qu'un vaisseau, si grand qu'on pourra le faire, peut être rendu vide de toutes les matières connues en la nature et qui tombent sous nos sens; et quelle force est nécessaire pour faire admettre ce vide : dédié à $M$. Pascal, conseiller du Roi en ses conseils d'État et privé, par le Sieur B. Pascal, son fils; le tout réduit en abrégé et donné par avance d'un plus grand traité sur le même sujet, Paris, Margot, 1647.

Pascal décrit huit expériences avec seringues, soufflets, tuyaux, siphons, de hauteurs, diamètres et formes variés, chargés de différents liquides. Toutes tendent au même but : prouver la possibilité du vide.

La troisième est celle qu'il réalise à Rouen, dans la cour de la verrerie, avec un tuyau de quarante-six pieds rempli de vin. Si on l'incline sur l'horizon, jusqu'à la hauteur de trente-deux pieds, «il se remplit entièrement $»$.

La sixième consiste à suspendre une seringue au fléau d'une balance, de telle sorte que sa pointe plonge dans une cuvette de mercure. On tire le piston qui entraîne le mercure en contact avec lui et l'on fait une première pesée; on soulève le piston jusqu'à ce qu'il laisse un espace vide entre le mercure et lui et l'on fait une seconde pesée. Si le vide apparent

41. [NdE - Omis par L. Rougier.] 
était rempli d'une matière sensible, son poids s'ajouterait à celui du mercure dans la seconde pesée et l'on devrait trouver une augmentation de poids. Or, on retrouve le même poids, et Pascal en conclut que l'espace vide en apparence l'est réellement. Ce faisant, il néglige la variation de la poussée de l'air dans les cas des deux pesées. L'air étant un fluide pesant, en vertu du principe d'Archimède, plus l'espace vide qui s'intercale entre le mercure et le piston est grand, plus est considérable le volume d'air déplacé par la seringue et plus la poussée de bas en haut exercée par l'atmosphère sur la seringue doit être forte. Avec une balance suffisamment sensible, avec un vide suffisamment grand, le résultat ne serait pas positif, comme Pascal en prête l'opinion à ses adversaires, il ne serait pas identique, comme l'affirme Pascal : il serait négatif.

Quelle est la portée de ces expériences? Les avis sont aussi opposés qu'il se peut. Écoutons d'abord le témoignage le plus favorable, celui de Pierre Duhem :

Que l'on relise, écrit M. Duhem, ces Nouvelles expériences. Il ne faut pas être grand clerc pour pressentir que l'auteur a des idées de derrière la tête et que ces idées sont précisément celles que Torricelli mandait à Ricci.

Pas plus que Torricelli, Pascal n'admet l'opinion de Galilée, d'après laquelle la suspension de l'eau en la pompe aspirante s'explique par une « repugnanza al vacuo »; pour rejeter cette opinion, Torricelli avait observé que le mercure montait à la même hauteur dans le tube, soit que l'espace vide fut grand, soit qu'il fut petit; et, Pascal s'applique, en chacune de ses expériences, à reconnaître qu'un grand vide ne fait pas plus d'effet qu'un petit.

Si l'ascension du vif-argent dans le tube barométrique s'explique par la pression de l'air, l'eau, quatorze fois plus légère que le mercure, doit s'élever quatorze fois plus que ce métal; Torricelli s'était borné à prévoir ce fait; pour le constater, Pascal ne recule pas devant des expériences pénibles et coûteuses.

Enfin, si la pression de l'air est la véritable raison des effets attribués à l'horreur du vide, les lois de l'hydrostatique veulent qu'un même liquide s'élève à la même hauteur verticale et dans un baromètre incliné à l'horizon; Pascal met tous ses soins à soumettre cette loi à des épreuves variées.

Assurément, celui qui conçoit et réalise de telles expériences possède la clé qui en ouvre l'interprétation; il sait que dans la théorie de Torricelli se trouve l'explication véritable des faits qu'il constate; s'il n'était Pascal, 
il donnerait tout aussitôt ces faits pour preuves très certaines de cette théorie.

Mais le sens critique de Pascal le met en garde contre cette hâtive conclusion : les Nouvelles expériences touchant le vide tendent toutes à favoriser la doctrine de Torricelli; mais il n'en est aucune qui condamne sans conteste la doctrine de Galilée, aucune qu'un disciple du Pisan ne puisse, avec quelque effort, revendiquer pour son parti. L'auteur va donc laisser en suspens ce que la logique n'a point tranché : il expose ses expériences sans en tirer, sur la nature de la force qui suspend le vif-argent dans le tube barométrique, des conclusions qu'elles ne suffisent pas à justifier ${ }^{42}$.

Cette opinion n'est pas partagée par Gaston Milhaud :

M. Duhem a dit à quel point les expériences de Pascal sont irréprochables en elles-mêmes, et je n'y reviendrais pas après lui si je ne croyais pas que, par derrière les représentations, il ait prêté à Pascal des pensées qu'il n'avait point encore. Pascal ne me paraît préoccupé dans toutes ces premières recherches que de la question du vide et du plein. Il s'efforce de réfuter, en variant ses procédés, ceux pour qui l'espace vide est plein de quelque matière subtile. En augmentant la grandeur de l'espace vide sans que la hauteur de la colonne en soit modifiée, il rejette l'hypothèse d'une action quelconque de cette prétendue matière sur le vif-argent. En pesant la seringue dont la pointe plonge dans le mercure et où celui-ci est monté à la suite du piston, d'abord quand le mercure est encore en contact avec le piston, puis quand le piston soulevé davantage a laissé un espace apparemment vide, il veut montrer qu'il n'est intervenu aucune matière pesante entre le mercure et le piston... Il ne s'est pas encore demandé ce que peut valoir l'explication de ses expériences par la pesanteur de l'air, il n'y songe peut-être pas, et, sans entrer dans le débat sur la cause de la suspension du mercure, sans formuler aucune théorie qui dépasse la portée de ces expériences, acceptant le langage courant de l'horreur du vide, il se propose uniquement de démontrer que cette horreur est limitée, c'est-à-dire que le vide est possible ${ }^{43}$.

42. P. Duhem, art. cit., Revue générale des sciences, 1906, p. 811.

43. Gaston Milhaud, «Pascal et les expériences du vide», ap. Revue scientifique, 1907, p. $773-774$. 
Ainsi, pour Pierre Duhem, les Expériences nouvelles s'interprètent naturellement en partant des lois de l'hydrostatique et malaisément en partant de la théorie de Galilée. Pour Gaston Milhaud, uniquement soucieux d'établir par ses expériences que l'espace apparemment vide l'est effectivement, Pascal ne songe nullement à leur interprétation théorique et emploie le langage courant de l'horreur du vide.

Selon M. Mathieu ${ }^{44}$, il en va tout autrement. Bien loin que ces expériences se présentent comme l'application d'une loi générale de l'équilibre des liqueurs, impliquant l'hypothèse de Torricelli, elles n'ont de sens qu'en fonction de la théorie de Galilée.

1) - Soit l'expérience du tube incliné. Implique-t-elle nécessairement, dans l'esprit de Pascal, la connaissance des lois de l'hydrostatique? Duhem, transportant, par un abus de la méthode de sympathie, les ressources de son savoir dans celui de l'auteur, lui prête une profondeur d'intention qu'il n'a pas. Pour Galilée et ses disciples, la hauteur de la colonne liquide, dont le poids contrebalançait l'horreur du vide, devait être mesurée verticalement et l'expérience du tube incliné, que tout le monde répétait, n'avait d'autre but que d'établir, qu'il n'est pas resté d'air dans le tuyau : quand on l'incline, le mercure vient frapper le sommet du tube avec un bruit sec. Les contemporains ne lui conféraient pas d'autre importance que celle-là. Cela est si vrai que Roberval la décrit dans sa première narration à Des Noyers, alors qu'il rejette l'explication par la colonne d'air. Une mauvaise gravure, huit fois reproduite dans les Reflexiones de Mersenne, montre un tube incliné auprès d'un tube vertical : or, Mersenne est tantôt pour, tantôt contre la colonne d'air, suivant qu'il subit l'influence de Descartes ou de Roberval.

2) - Soit maintenant la sixième expérience. Elle prouve que Pascal est si éloigné de penser à la pression atmosphérique, qu'il ne croit pas encore à la pesanteur de l'air.

Pascal affirme avoir pesé une seringue dans laquelle il faisait varier les dimensions de l'espace vide et avoir trouvé toujours le même poids. C'est une expérience absurde, mal faite et mal interprétée; théoriquement, le poids de la seringue doit varier avec les dimensions du vide et, si Pascal enregistre ce résultat sans le discuter, c'est qu'il ne croit pas à la pesanteur de l'air ${ }^{45}$.

44. R.P., 1906, p. 587.

45. Mathieu, «Pascal et l'expérience du Puy-de-Dôme», ap. Revue de Paris, $1^{\text {er }}$ mars 1907, p. 194. 
Il est bien vrai, comme le remarque Gaston Milhaud, que Pascal est obnubilé par l'unique pensée de prouver la réalité du vide, c'està-dire d'établir qu'aucune matière n'occupe l'espace laissé vide par le piston. Mais eût-il été en possession, dès cette époque, des lois de l'hydrostatique, eût-il admis que l'air est un fluide pesant auquel le principe d'Archimède s'applique, comment n'eût-il pas été surpris du résultat obtenu, puisque le poids de la seringue, à mesure qu'on élève le piston, doit diminuer en raison du plus grand volume d'air déplacé?

Pierre Duhem, à la décharge de Pascal, déclare que la poussée de l'air, dont Pascal ne parle pas, est négligeable en de telles circonstances. Rapprochant l'expérience de la seringue d'un raisonnement de Mersenne qui s'applique à tout autre chose, il déclare :

L'expérience incriminée est absolument exacte : elle apporte à l'expérience de Torricelli un complément de très grande importance; tous les traités de physique en donnent l'explication; elle est le fondement du baromètre [statique?] imaginé en 1670, par Samuel Morland pour déterminer la pression atmosphérique au moyen d'une pesée, et du baromètre enregistreur, composé en 1782 par Magellan ${ }^{46}$.

Mais une première difficulté se présente. Si l'expérience de la seringue constitue pour Pascal un si beau titre de gloire, comment expliquer qu'il l'ait soigneusement biffée de ses Traités posthumes, alors que, précisément, [phrase manquante $][\ldots]$ du «langage » de Galilée à celui de Torricelli? En second lieu, Duhem rapproche indûment deux expériences fort distinctes : dans celle du baromètre statique, il y a variation de poids, ce qui permet de déterminer la pression atmosphérique, dans celle de Pascal, la variation du vide n'entraîne pas une variation de poids. Enfin, par quel étrange abus de langage, Duhem soutient-il que l'expérience de la seringue est « absolument exacte»! Si Pascal, dans les expériences qu'il propose pour établir l'existence du vide, ne soupçonne pas la volatilisation spontanée des liquides dans le vide, dans l'expérience de la seringue, où il croit l'espace vide, il aurait dû penser que la variation du volume vide entraîne la variation de la poussée de l'air due au principe d'Archimède, qu'une balance plus sensible eût révélée. Son résultat est, à tout le moins, théoriquement inexact. Plus tard, dans ses Traités posthumes, il reconnaîtra sa bévue. Il verra, ce qu'il ne voit pas ici, que son expérience est «absurde» et il la retranchera de la suite des autres.

46. Art. cit., p. 812. 
Nous serions tentés de nous rallier à l'opinion moyenne de Gaston Milhaud, toutes réserves faites sur l'exactitude des expériences de Pascal, que l'auteur semble admettre sous la suggestion de Duhem. Pascal, à cette époque, n'ayant nullement en vue de rechercher la cause de la suspension des liquides dans les tubes barométriques, ne songe et n'a pas à songer à la doctrine de Torricelli. Malheureusement, cette attitude, qui se définit par une suspension de jugement, ne peut avoir été celle de Pascal. Une des maximes qu'il déduisit de ces expériences vient formellement témoigner que la doctrine galiléenne de l'horreur limitée du vide était la seule à laquelle il pouvait penser demander la clé explicative de ses expériences nouvelles. Il n'est que de lire les conclusions de la première partie de l'Abrégé :

Desquelles expériences on déduit manifestement ces maximes :

1. Que tous les corps ont de la répugnance à se séparer l'un de l'autre, et à admettre du vide dans leur intervalle, c'est-à-dire que la nature abhorre le vide.

2. Que cette horreur ou répugnance qu'ont tous les corps n'est pas plus grande pour admettre un grand vide qu'un petit...

3. Que la force de cette horreur est limitée, et pareille à celle avec laquelle l'eau d'une certaine hauteur, qui à peu près de trente et un pieds, tend à couler en bas.

4. Que les corps qui bornent ce vide ont inclination à le remplir.

5. Que cette inclination n'est pas plus forte pour remplir un grand vide [apparent] qu'un petit.

6. Que la force de cette inclination est limitée et toujours ${ }^{47}$ pareille à celle avec laquelle l'eau d'une certaine hauteur, qui est environ trente et un pieds, tend à couler en bas.

Si la sixième expérience semble exclure la pesanteur de l'air, à coup sûr, la sixième maxime rejette catégoriquement l'explication par la pression atmosphérique. Qu'énonce-t-elle, en effet, sinon que la répugnance qu'ont les corps à se séparer les uns des autres a une grandeur finie et que cette grandeur est constante, à savoir qu'elle est égale à la force avec laquelle l'eau, élevée à trente et un pieds, tend à couler en bas. Admettre que

47. [NdE - Souligné par L. Rougier.] 
l'horreur du vide est une grandeur constante, c'est exclure la colonne d'air dont la pression varie avec l'altitude et si l'auteur s'avise de faire l'expérience à des altitudes différentes, c'est qu'il escompte un résultat négatif. Aurait-on la moindre envie d'en douter, il suffirait de lire le commentaire que Pascal a fourni de cette sixième maxime dans la conclusion de ses Traités posthumes.

Galilée déclare, dans ses Dialogues, qu'il a appris des fontainiers d'Italie, que les pompes n'élèvent l'eau que jusqu'à une certaine hauteur; ensuite de quoi il l'éprouva lui-même; et d'autres ensuite en firent l'épreuve en Italie et depuis en France avec du vif-argent, avec plus de commodité, mais qui ne montraient que la même chose en plusieurs manières différentes...

Mais toutes ces expériences ne suffirent pas pour montrer que l'air produit ces effets, parce qu'encore elles nous eussent tirés d'une erreur, elles nous laissaient dans une autre : car ON apprit bien par toutes les expériences que l'eau ne s'élève que jusqu'à une certaine hauteur, mais on n'apprit pas qu'elle s'élevât PLUS HAUT DANS LES LIEUX PROFONDS : ON PENSAIT, AU CONTRAIRE, QU'ELLE S'ÉLEVAIT TOUJOURS À LA MÊME HAUTEUR, QU'ELLE ÉTAIT INVARIABLE EN TOUS LES LIEUX DU MONDE; ET COMME ON NE PENSAIT POINT À LA PESANTEUR DE L'AIR, $\mathrm{ON}^{48}$ s'imagina que la nature de la pompe est telle qu'elle élève l'eau à une certaine hauteur limitée, et puis plus. Aussi, Galilée la considéra comme la hauteur naturelle de la pompe, il l'appella LA ALTESSA LIMITATISSIMA.

La dernière maxime reproduit, elle aussi, la pensée de Galilée :

VII - Qu'une force plus grande, de si peu que l'on voudra, que celle avec laquelle l'eau de la hauteur de trente et un pieds tend à couler bas, suffît pour faire admettre du vide, et même si grand que l'on voudra, POURVU QU'IL N'Y AIT POINT D'AUTRE OBSTACLE À LEUR SÉPARATION, NI À LEUR ÉLOIGNEMENT, QUE L'HORREUR QUE LA NATURE A POUR LE VIDE ${ }^{49}$.

48. [NdE - Souligné par L. Rougier.]

49. [NdE - Souligné par L. Rougier. La citation n'est pas tout à fait exacte. Pascal en fait a écrit : « ... si grand que l'on voudra ; c'est-à-dire pour faire désunir les corps 
Dans la deuxième partie de son Abrégé, Pascal rapporte «les conséquences de ces expériences, touchant la matière qui peut remplir l'espace vide en apparence ».

Il n'est pas rempli de « l'air extérieur... entré par les pores du verre $»$; ni de l'air « que quelques philosophes disent être enfermé par les pores de tous les corps » et par la suite « de la liqueur qui remplit le tuyau »; ni «d'un grain d'air imperceptible » entré accidentellement; ni des vapeurs du vif-argent ou de l'eau; ni des « esprits» de ces liqueurs; ni d'un «air plus subtil »; bref, ce vide apparent «n'est rempli d'aucune des matières qui tombent sous aucun sens ».

Mon sentiment sera donc, jusqu'à ce qu'on m'ait démontré l'existence d'une matière qui le remplisse, qu'il est véritablement vide et destitué de toute matière ${ }^{50}$.

C'est pourquoi je dirai du vide véritable ce que j'ai montré du vide apparent, et je tiendrai pour vraies les maximes posées ci-dessus, et énoncées du vide absolu comme elles l'ont été de l'apparent.

Que conclure? Premièrement, les expériences de Pascal n'ont pas cette absolue exactitude que leur confère Duhem. Ce dernier sait fort bien que le vide apparent est rempli de vapeur de mercure et que la seringue qui déplace d'autant plus d'air qu'elle contient plus de vide, subit la poussée archimédienne de l'air. On se demande alors comment il oublie complètement ce qu'il sait si pertinemment? Tout ce qu'un savant, averti comme lui, peut actuellement penser sur la portée physique des Expériences nouvelles, c'est que la tension maxima du mercure, à la température où Pascal opérait, était négligeable, et que la sensibilité de la balance qu'il employait était inférieure à la variation de la poussée de l'air. En second lieu, Pascal ne conçoit pas d'autre cause possible de la suspension de la colonne liquide que l'horreur limitée du vide.

Qu'on relise, en dehors de tout souci de justification, l'Abrégé. On y verra que, conformément au titre, les expériences touchant le vide qu'on y décrit tendent toutes à démontrer la possibilité du vide et à établir que « la force nécessaire pour faire admettre ce vide, à savoir l'horreur du

d'un si grand intervalle que l'on voudra, pourvu qu'il n'y ait point d'autre obstacle à leur séparation, ni à leur éloignement que l'horreur que la nature a pour ce vide apparent ». En outre, cette «dernière maxime» provient de l'Abrégé, et non des Traités posthumes de Pascal, comme on serait peut-être tenté de le penser.]

50. [NdE - Souligné par L. Rougier.] 
vide, est une force limitée et constante ». Prêter à Pascal, comme arrièrepensée, la théorie de Torricelli, sous prétexte que cette théorie est seule capable de justifier théoriquement ses expériences, alors qu'elle donne à la suspension des liquides une cause essentiellement variable, la pression atmosphérique, c'est pur parti pris. Le maître pisan réalise son expérience en vue d'établir un baromètre, c'est-à-dire de repérer par les variations de la colonne de mercure les variations de la pression atmosphérique. Dans l'Abrégé, rien ne suggère l'idée du baromètre, et la doctrine qui s'y déploie exclut la possibilité même de cet instrument. Pour se rallier à la thèse de Duhem, il faudrait préjudiciellement expliquer deux choses : $\left.1^{\circ}\right)$ pourquoi, si l'expérience de la seringue est d'une « admirable précision » et le «fondement du baromètre statique », Pascal a biffé une expérience qui constituait un si beau titre de gloire de ses Traités posthumes. $2^{\circ}$ ) Comment peut-on, ayant l'hypothèse de Torricelli derrière la tête, rendre compte de la sixième maxime de l'Abrégé?

\section{6 - Les témoignages de Guiffart, de Roberval, de Mersenne, de Dominicy et de Descartes}

À l'aveu de Pascal, consigné explicitement dans l'Abrégé, se joignent des témoignages extérieurs. Si Pierius entreprend Pascal pour défendre contre lui la thèse péripatéticienne de l'impossibilité du vide, c'est pour défendre Pascal contre les entreprises de Pierius, que le médecin Pierre Guiffart, témoin des expériences et auditeur des conférences de Rouen, rédigea son Discours du vuide sur les expériences de $M$. Pascal et de M. Pierius, achevé d'imprimer le 29 août 1647. Cette longue dissertation est une réfutation en règle du livre de Pierius et tend à dégager la moralité des expériences de Rouen. Elle constitue une reprise pure et simple de la théorie de Galilée. L'auteur tend à démontrer la possibilité du vide qui « eut l'honneur d'assister à la naissance du monde » et l'horreur limitée qu'il inspire à la nature. À ceux qui prétendent que les éléments s'effondreraient si un vide se produisait, en sorte que le feu tomberait sur l'air, et l'air sur l'eau, il répond que l'air, à l'instar de tous les corps célestes, n'est ni lourd, ni léger; il ne peut ni monter, ni descendre, mais tourner seulement.

Puisqu'il entreprend de défendre Pascal contre Pierius, il est logique de penser que Guiffart nous expose fidèlement les directives doctrinales qui se dégageaient des leçons de Pascal. À ces auditeurs, celui-ci était bel et bien apparu comme un disciple de Galilée. Peut-on aller plus loin et conclure, de ce que Guiffart s'inscrit contre la pesanteur de l'air, que Pascal en 1646 faisait profession de la rejeter explicitement? Dans ce 
cas, l'interprétation que Mathieu donne de l'expérience de la seringue serait vérifiée. Mais Galilée, dans les Discorso, admet la gravité de l'air dont il cherche à déterminer la densité : comment Guiffart et Pascal pourraient-ils être réputés alors disciples du Florentin? Il faut se souvenir que Galilée, en 1615, affirmait encore, contre Vicenzo di Grazia, que l'air normal, non condensé, n'est ni pesant ni léger. Tout se passerait, selon cette conjoncture, comme si tous deux avaient lu la première journée des Dialogues, où ils ont appris que l'horreur du vide est limitée et égale au poids d'une colonne d'eau de trente-deux pieds, mais sans être allés plus avant dans leur lecture.

Comme il est bien peu vraisemblable que Pascal se soit inspiré de Guiffart, il faut admettre que c'est Guiffart qui, en août 1647, est l'écho de Pascal, dont l'attitude, quelque six mois plus tard, n'était pas modifiée. On ne s'étonne plus, alors, de trouver, dans la pauvre dissertation de physique du médecin, plusieurs idées qui, au style près, se retrouvent dans le Discours sur le vide. L'autorité des Anciens n'est souveraine que « lorsque le fil de notre raison se trouve trop court pour nous conduire dans le labyrinthe des difficultés ». Cependant, lorsqu'un esprit curieux, mais désintéressé, peut en venir à bout, «ni le respect de l'Antiquité, ni l'aversion de la nouveauté ne doivent aucunement empêcher de prononcer $\gg$. Tel est le cas des expériences de Pascal.

Ceux qui sont philosophes ne les peuvent voir sans admiration, et ceux qui ne le sont pas le deviennent en les considérant.

Les aphorismes, dont Pascal composera son Discours sur le vide, ne les aurait-il pas proférés lors de ses conférences publiques de Rouen, dans l'action de la découverte?

Le second témoin à produire est Roberval en personne, dans l'épître qu'il adresse le 20 septembre 1647 à Des Noyers, où il relate, en observateur averti, les expériences de Pascal, y joignant les siennes propres et ses réflexions. Qu'y lisons-nous? Les expériences de Rouen ont pour objet d'écarter deux hypothèses plausibles concernant le vacuum : la chambre barométrique est pleine d'air raréfié; et elle est remplie de vapeurs de mercure, pour conclure que le vide apparent n'est rempli d'aucune matière sensible. Roberval incline pour sa part à y croire, sans oser absolument se prononcer :

On ne peut rien conclure de certain au sujet de cet espace, qui apparaît comme étant vide, dans lequel des corps peuvent se mouvoir et par lequel se transmet la lumière avec les couleurs. 
Pour être en droit d'être plus catégorique, il faudrait réaliser des expériences avec des animaux et voir s'ils peuvent vivre et surtout voler dans la chambre barométrique, auquel cas on devrait conclure à la présence de l'air. Or, que pensait le même Roberval de l'hypothèse de Torricelli? Le dernier chapitre des Reflexiones de Mersenne nous le montre accumulant les objections contre la colonne d'air et soutenant une théorie de l'attraction. Ainsi, Roberval, confident des préoccupations de Pascal, témoin de ses expériences, incline à cette époque au vide et rejette absolument la colonne d'air.

Un troisième témoin est le père Mersenne. Dans la seconde Préface de ses Reflexiones, rédigée dans les derniers jours de septembre et les premiers d'octobre 1647, il déclare que, décidément, la colonne d'air est une absurdité; la seule explication plausible est l'attraction. Il espère bien que le jeune Pascal l'établira solidement dans le Traité auquel il travaille présentement. Ainsi, Mersenne, au début d'octobre 1647, tenait Pascal pour un adversaire de l'hypothèse de Torricelli.

Plus proche encore de la date de la parution de l'Abrégé, est l'aveu d'un personnage fort énigmatique, qui signe Dominicy. Il fit paraître à Paris, dans le courant du mois de novembre, une brochure intitulée : Observation touchant le vuide faite pour la première fois en France; contenue en une lettre écrite à $M$. Chanut, résident pour Sa Majesté en Suède, par M. Petit, intendant des fortifications, le 10 novembre 1646. Avec le discours qui a été imprimé en Pologne sur le même sujet, en juillet $164 \%$.

La lettre de Petit à Chanut est celle du mois de novembre 1647. Le discours imprimé en Pologne n'est autre que la Demonstratio ocularis du P. Magni. Le privilège est daté du 12 novembre 1647.

Dans la dédicace à Séguier, l'éditeur de la brochure, tout en se défendant d'agir au nom de Petit, se dit indigné qu'on ait publié ailleurs les observations de celui-ci «sans lui en rendre gloire ». S'il publie sa lettre, en dépit de l'auteur qui s'y est toujours opposé, c'est «parce qu'elle fait partie de l'histoire de cette nouveauté et que l'auteur est comme le premier mobile qui donne le branle à tout ce qui s'est fait et dit depuis en cette matière $»$.

Dans la Préface, il accuse fort inexactement Magni de s'être vanté d'avoir été le premier à faire l'expérience du vide, alors qu'il n'avait réalisé que ce qui avait été « écrit » et fait en France «neuf mois auparavant, pendant lesquels on en pourrait porter la nouvelle en Chine», et sur les indications d'un gentilhomme français qui était parti pour la Pologne, après avoir assisté aux expériences de Rouen. D'ailleurs, Pascal, depuis, a fort enchéri sur ces premières observations. 
Dominicy conteste formellement à Torricelli — qui vient de mourir — «la gloire de l'invention» pour la donner à Galilée. Dans ses Discours et démonstrations mathématiques, celui-ci a, le premier, enseigné qu'on peut faire le vide avec une seringue et qu'on ne peut faire monter l'eau plus haut que trente-deux pieds. Pour un partisan convaincu de l'horreur limitée du vide, Galilée est bien l'initiateur dont Pascal est le digne émule. Quant à Torricelli, il s'est simplement fourvoyé : il n'y a pas lieu d'en tenir compte.

Quel est donc ce Dominicy? Son nom ne se retrouve nulle part ailleurs, dit M. Mathieu. Il n'agit pas à l'instigation de Petit, mais plutôt à celle de Pascal, qui lui a communiqué une copie, conservée par lui, de la lettre de Petit et qui l'a autorisé à la publier. Il ne donne ni le nom du « gentilhomme français », parti de Rouen pour la Pologne, qui aurait instruit Magni, ni la preuve que Magni l'ait rencontré. Tout laisse entendre que c'est un ami de Pascal qui plaide sa cause à propos de celle de Petit ; qui sait même, un pseudonyme de Pascal, insinue Mathieu. Nous verrons Pascal, dans la lettre à Ribeyre, tenir le langage même de Dominicy. En tous cas, le but de l'auteur est clair. En écartant Torricelli, coupable de s'être mépris, et Magni, coupable d'avoir plagié, pour ne conserver que Galilée, inventeur de l'horreur limitée du vide, Petit, « qui a donné le branle » et Pascal le Jeune, « qui a beaucoup enchéri par-dessus ces [premières] observations », Dominicy montre que l'auteur de l'Abrégé procède directement de Galilée et qu'il est, après lui, l'artisan par excellence de cette théorie de l'horreur limitée du vide dont il assurera bientôt le triomphe définitif.

Sans qu'il soit besoin de faire de Dominicy autre chose qu'un ami de Pascal, sa publication, venant corroborer les témoignages de Guiffart, Roberval et Mersenne, prouve bien, comme il se dégage de la lecture de l'Abrégé, que toute l'ambition de Pascal en novembre 1647 était de faire figure de continuateur de Galilée.

Un plus tardif et dernier témoignage est celui de Descartes, dont l'on sait combien la mémoire est fidèle. Tardivement informé par Carcavi du succès de l'expérience du Puy-de-Dôme, il le remercie le 17 août 1649 de lui en avoir mandé le résultat.

J'avois quelque intérest à le sçavoir, à cause que c'est moy qui l'avois prié il y a deux ans de la vouloir faire, et je l'avois assuré du succès comme étant entièrement conforme à mes Principes, sans quoy il n'eut garde d'y penser, à cause qu'il estoit d'opinion contraire ${ }^{51}$. Et, pour ce qu'il m'a cy-devant

51. [NdE - Souligné par L. Rougier.] 
envoyé un petit Imprimé (l'Abrégé) où il décrivoit ses premières expériences touchant le vuide, et promettoit de réfuter ma matière subtile, si vous le voyez je serois bien aise qu'il sceut que j'attends encore cette réfutation, et que je le recevray en très bonne part, comme j'ay toujours receu les objections qui m'ont esté faites sans calomni ${ }^{52} \ldots$

Les conversations de Descartes avec Pascal sont des 23 et 24 septembre 1647. L'impression laissée par Pascal sur l'esprit de Descartes est qu'il était adversaire de la colonne d'air et contestait la nécessité s'une expérience telle que celle du Puy-de-Dôme. Ces deux éventualités sont exclues par la sixième maxime de l'Abrégé.

52. Adam, V, 369. [NdE - Si l'on suit les abréviations de L. Rougier, il faut lire : D. A., V. Les pages exactes sont les pages 391-392.] 